Letter to Editor

\title{
Correlation between NT pro-b-type Natriuretic peptide and left ventricular ejection fraction in children presenting with Dyspnea- a prospective cohort study
}

\author{
Aswathy Rajan ${ }^{1}$, Shriyan Ashvij ${ }^{2}$, Prem Alva ${ }^{3}$, Santosh T Soans ${ }^{4}$ \\ ${ }^{1}$ Dr. Aswathy Rajan, Assistant Professor, Department of Pediatrics, A. J. Institute of Medical Sciences, Mangalore, \\ ${ }^{2}$ Dr. Shriyan Ashvij, Assistant Professor, Department of Pediatrics, A. J. Institute of Medical Sciences, Mangalore, \\ ${ }^{3}$ Dr. Prem Alva, Consultant Pediatric Cardiologist, Department of Pediatric Cardiology, A. J. Hospital and Research \\ Centre, Mangalore, ${ }^{4}$ Dr. Santosh T Soans, - Professor and HOD, Department of Pediatrics, A. J. Institute of Medical \\ Sciences, Mangalore, Karnataka, India.
}

Corresponding Author: Dr. Shriyan Ashvij, Assistant Professor, Department of Pediatrics, A. J. Institute of Medical Sciences, Mangalore, Karnataka, India. E-mail: ashvijs@yahoo.co.in

\begin{abstract}
Introduction: Simple screening tests exist for congenital heart diseases but the same cannot be said for cardiac failure in children. Although echocardiography is considered the gold standard for the detection of left ventricular dysfunction, it is relatively expensive and is not often readily available. We aim to prove that NT pro-B-type natriuretic peptide levels may be useful in ruling out the diagnosis of heart failure in children presenting to the emergency room with dyspnea by studying the relation between left ventricular ejection fraction and NT pro-BNP. Methods: Seventy four patients presenting to the emergency department with dyspnea and fulfilling the modified Ross' criteria for heart failure were included in the study. NT pro-BNP levels were measured for all the patients and a two-dimensional echocardiographic study was performed for them. Statistical analyses were performed using chi square test for independent samples and Pearson correlation tests. Results: A significant inverse correlation was observed between NT pro-BNP levels and left ventricular ejection fraction $(\mathrm{r}=-0.789, \mathrm{p}=0.003)$. Conclusion: NT pro-BNP assay appears to hold promise as a potent and cost effective test of choice for acutely dyspneic children, which could replace chest x-ray and echocardiography as first investigation in such patients in urgent care settings.
\end{abstract}

Keywords: NT pro-BNP, left ventricular ejection fraction, heart failure

\section{Introduction}

Heart failure is estimated to affect 12,000 to 35,000 children below 19 years of age in the United States each year. The presentation of pediatric heart failure is diverse because of the numerous underlying cardiac etiologies and varying clinical settings [1]. The recognition of heart disease in children can be challenging as children often have a limited repertoire of presenting signs and symptoms. Many cardiac disease states can mimic the more common illnesses of childhood, such as bronchiolitis, reactive airway disease, and sepsis. The diagnosis of heart disease can be especially difficult when children present at institutions that do not specialize in pediatric health care and are without ready access to pediatric echocardiography $[2,3]$.

Manuscript received: $4^{\text {th }}$ November 2018

Reviewed: $10^{\text {th }}$ November 2018

Author Corrected: $15^{\text {th }}$ November 2018

Accepted for Publication: $19^{\text {th }}$ November 2018
Pediatric patients with cardiac disease that results in profound cyanosis or a loud murmur usually have rapid recognition and diagnosis as a result of the prominent physical findings. Many of these presenting signs and symptoms also present in other common diseases of childhood, most notably in viral illnesses. A reliable marker of heart disease in children would be of great benefit to those who practice pediatric health care and to the children in their care [2].

$\mathrm{N}$-terminal pro-Brain-natriuretic peptide (NT pro-BNP) is an excellent marker of heart failure in adults. Serum levels can help differentiate dyspnea caused by respiratory problems from heart failure, correlate with the severity of left ventricular dysfunction and functional status, predict morbidity and mortality, and can guide medical treatment. However, the value of measuring serum levels of NT pro-BNP in children with 
Letter to Editor

heart failure still needs further study before it can be recommended for clinical use [4]. Although echocardiography is considered the gold standard for the detection of left ventricular dysfunction, it is relatively expensive, is not often readily available and may not always be diagnostic in acute presentation and user dependent [4].

Hence, in this study we try to prove that when used in conjunction with other clinical information, NT proBNP levels may be useful in establishing or ruling out the diagnosis of heart failure in patients with acute dyspnea.

NT pro-BNP assay appears to hold promise as a potent and cost effective test of choice for acutely dyspneic patients, which could replace chest x-ray and echocardiography as first investigation in such patients in urgent care settings. In this study, we hypothesize that changes in NT-proBNP serum levels are associated with changes in echocardiographic indices of $\mathrm{LV}$ systolic function [4].

\section{Methodology}

Type of Study- A prospective cohort study.

Place of study- The study was conducted on 96 children presenting to the emergency room from November 2011 to October 2013 at A. J. Institute of Medical Sciences, Mangalore. Ethical committee clearance was taken from the institutional ethics committee for the same.

Inclusion criteria

All children aged 7 days to 14 years who presented with acute dyspnea were included in the study. Children were included after screening using Ross' criteria for heart failure. Ross' criteria was used due to its usefulness as a standard descriptor of respiratory symptoms in children.

Exclusion criteria- Children who were diagnosed with sepsis, renal dysfunction defined as serum creatinine $>$ $1 \mathrm{mg} / \mathrm{dl}$ or that which requires therapy, other problems that were not considered to be of cardiac or pulmonary etiology, anxiety or psychological stress, gastroesophageal reflux disease, allergic reaction or cirrhosis, obvious traumatic cause of dyspnoea.
Data and sample collection- All the patients were subjected to clinical history taking, a physical examination, electrocardiography, pulse oximetry, routine blood investigations, NT pro-BNP levels, chest radiography, and echocardiography.

Routine blood investigation were analysed including complete blood count, C-reactive protein, erythrocyte sedimentation rate, renal function tests and blood culture. For NT pro-BNP assay, $5 \mathrm{ml}$ specimen of venous blood was collected in EDTA tubes and analysed by chemiluminescent immunoassay. The minimum value read by the triage kit is $5 \mathrm{pg} / \mathrm{ml}$.

The diagnosis of CHF was based on independent confirmation of the cardiologist and results of echocardiography showing left ventricular dysfunction. Children with left ventricular ejection fraction of less than 50 were considered to be having cardiac failure.

The following diagnostic tools were utilized to classify a patient as having pulmonary disease and to subdivide them as type of pulmonary disease: A chest X-ray without signs of heart enlargement or pulmonary venous hypertension or a chest X-ray with signs of lung disease or reactive airway, normal heart function as seen by echocardiography, a positive response to treatment with steroids, nebulizers or antibiotics in the emergency department or hospital, no admissions for CHF over the next 30 days.

Data analysis- Two cohorts of children were evaluated. The cardiac cohort consisted of children who were diagnosed by the cardiologist to have cardiac failure recognized by left ventricular dysfunction on echocardiograph. The non-cardiac cohort consisted of the remaining children who were diagnosed with respiratory illness and no cardiac illness as confirmed by the cardiologist.

Statistical analysis- Data was analyses using SPSS version 17.0 statistical software. Spearman's Rank correlation coefficient was calculated for Spearman's test was applied for analysing relation between NT-pro BNP levels and left ventricular ejection fraction. Pearson chi square test was used for analysing categorical data. A probability of $<0.05$ was taken as significant.

\section{Results}

A total of 98 children presented to the emergency room with respiratory distress and fulfilling Ross' criteria during the study period and were enrolled for the study. 22 patients were excluded after the routine blood investigations. 12 were diagnosed with sepsis, 3 had distress neither due to cardiac or pulmonary etiology, 5 had traumatic cause of dyspnea, 1 had an allergic reaction and 1 child had a high creatinine value. The remaining 76 were included in the study. 19 children 


\section{Letter to Editor}

were diagnosed with a cardiac disease in failure, 57 were diagnosed with respiratory pathology. The minimum age included was 2.00 and the maximum age was 350.00 months which corresponds to a mean of 40.98 months with a standard deviation of 72.79 months.

The NT pro-BNP values were estimated in all the children. The mean NT pro-BNP level in the cardiac cohort was found to be $959.39 \pm 220.22 \mathrm{pg} / \mathrm{ml}$ while in the non-cardiac cohort it was found to be $191.79 \pm 244.79 \mathrm{pg} / \mathrm{ml}$. While comparing mean NT pro-BNP levels in both cohorts, the level was found to be significantly higher in the cardiac cohort than the non-cardiac cohort $(\mathrm{p}=0.001)$ as shown in Table 1 .

Table-1: Levels of NT pro-BNP in cardiac and non-cardiac cohort

\begin{tabular}{|c|c|c|c|c|c|}
\hline Group & $\mathbf{N}$ & $\begin{array}{c}\text { Mean } \\
(\mathbf{p g} / \mathbf{m l})\end{array}$ & Standard Deviation & Minimum & Maximum \\
\hline Cardiac & 19 & 959.39 & 220.22 & 85.00 & 1474.00 \\
\hline Respiratory & 57 & 191.79 & 244.79 & 42.00 & 1177.00 \\
\hline
\end{tabular}

$\mathrm{f}=0.6488, \mathrm{p}$ value is $<0.001$

On echocardiographic evaluation, all the children included in the cardiac cohort had NT pro-BNP values $\geq 500 \mathrm{pg} / \mathrm{ml}$. Among the children who had LVEF $\geq 50 \%(\mathrm{n}=57), 50(87.7 \%)$ had a NT pro-BNP level $<500 \mathrm{pg} / \mathrm{ml}, 6(10.5 \%) \mathrm{had}$ a value between 500 and $1000 \mathrm{pg} / \mathrm{ml}$ and $1(1.8 \%)$ of them had a value $\geq 1000 \mathrm{pg} / \mathrm{ml}$. The chi-square was 22.713 and $\mathrm{p}$ value of $<0.001$ was obtained (Table 2 ).

Table-2: Relation of NT pro-BNP level to left ventricular ejection fraction

\begin{tabular}{|c|c|c|c|c|}
\hline \multirow{2}{*}{ NT pro-BNP level } & \multicolumn{2}{|c|}{ LVEF $<\mathbf{5 0} \%$} & \multicolumn{2}{c|}{ LVEF $\geq \mathbf{5 0 \%}$ (n) } \\
\cline { 2 - 5 } & $\mathbf{N}$ & $\mathbf{\%}$ & $\mathbf{n}$ & \% \\
\hline$<500 \mathrm{pg} / \mathrm{dl}$ & 0 & 0 & 60 & 10.5 \\
\hline $500-1000 \mathrm{pg} / \mathrm{dl}$ & 10 & 52.6 & 1 & 1.8 \\
\hline$>=1000 \mathrm{pg} / \mathrm{dl}$ & 9 & 47.4 & $\mathbf{5 7}$ & $\mathbf{1 0 0}$ \\
\hline Total & $\mathbf{1 9}$ & $\mathbf{1 0 0}$ & 67.7 \\
\hline
\end{tabular}

chi-square $=22.713, \mathrm{p}$ value $-<0.001$

A significant negative correlation was obtained between LVEF and NT pro-BNP such that as the LVEF decreases, the NT pro-BNP level was found to be increasing $(r=-0.789, \mathrm{p}=0.001)$ (Figure 1).

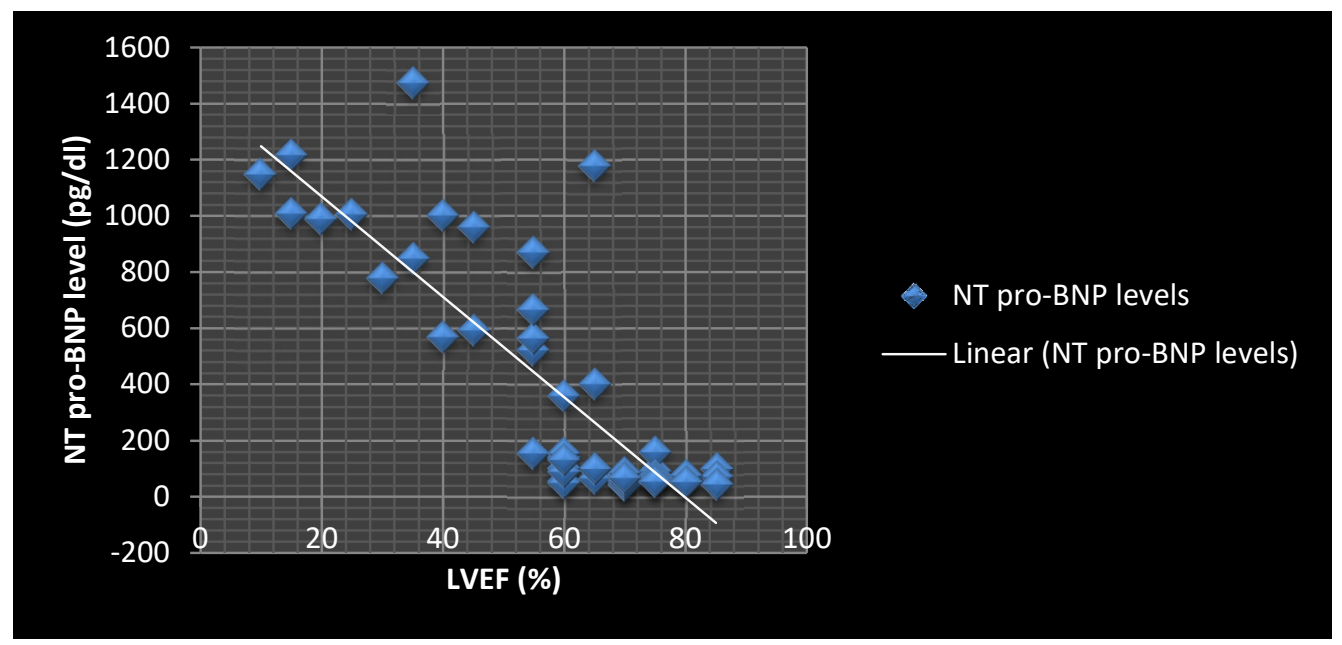

Figure-1: Correlation between LVEF and NT pro-BNP Correlation Coefficient - 0.789 


\section{Discussion}

In healthy infants and children, levels of B-type natriuretic peptide (BNP) and the N-terminal segment of its pro-hormone (NT pro-BNP) are elevated in the first few days after birth. Thereafter, their levels decrease and remain relatively constant throughout childhood. Infants and children with heart disease that causes significant pressure or volume overload of the right or the left ventricle have elevated BNP and NTpro BNP levels [5]. The importance of the NT pro-BNP level in the diagnosis and evaluation of heart failure has been widely accepted in adults. The NT pro-BNP level has been observed to increase with the abnormally high intra-ventricular pressure that is associated with heart failure, and the level is positively correlated with the severity of heart failure [6].

Studies show that a normal NT pro-BNP level has a high negative predictive value for heart failure. Natriuretic peptide levels in plasma correlated well with biventricular volume. The left ventricle was considered to be the main source of secreted natriuretic peptides in the patients with a pulmonary-to-systemic flow ratio greater than 2 [7]. Elevation of BNP/pro-B-type NP are seen due to long term exposure of right heart or left heart to volume and pressure overload. These elevations are especially seen with diseases that causes left ventricular volume overload when compared to right ventricular volume or pressure overload. Further more, when comparing pediatric populations with complex CHD vs simple cardiac defects (ASD, VSD or PDA), on average, complex defects tend to have higher concentrations [8].

An accepted normal level of NT pro-BNP is considered to be $<300 \mathrm{pg} / \mathrm{ml}$ in children with non-cardiogenic dyspnea. Lin et al, in their study, showed an increased NT-proBNP level in $95 \%$ of the 80 children diagnosed with heart failure according to the modified Ross criteria. Moreover, there were also significant differences in the NT-proBNP levels among cases of mild, moderate and severe heart failure [6].

According to a review done by Robert Ross in 2012, NT pro-BNP values for classifying heart failures ranges from $450-1700 \mathrm{pg} / \mathrm{ml}$ in various age groups.[9] However, in our study, all the children in the cardiac cohort had high values of NT pro-BNP levels above 500 $\mathrm{pg} / \mathrm{ml}$ out of which $44 \%$ of them had a value above $1000 \mathrm{pg} / \mathrm{ml}$. Rusconi et al determined that an NTproBNP value $>1,000 \mathrm{pg} / \mathrm{mL}$ identifies more symptomatic patients. Their results indicated that increase in NT pro-BNP serum levels are associated with echocardiographic signs of failure (lower LVEF, lower left ventricular shortening fraction, higher left ventricular diastolic diameter, higher left ventricular systolic diameter) and increased odds of being in NYHA/ Ross classes III or IV [4].

A study done by Zhou et al between 2009 and 2012 with the mean age of their subjects as $3.49+-0.23$ years showed that the levels of NT pro-BNP reflected the severity of heart failure and it is significantly upregulated in the condition. Their data reveals significantly higher values of NT pro-BNP in children with heart disease as compared to those with pneumonia. The positive rate of NT-proBNP was up to $100 \%$ in children with heart function above class II, which suggested a positive correlation between NTproBNP level, making it an imperative index for the clinical diagnosis of heart failure and a suitable tool for prognostic evaluation [10].

A study done by Das et al compared the value of BNP in patients with diagnosis of respiratory distress due to primary respiratory illness or infection vs new diagnosis of CHD in an emergency room setting. A mean BNP value of $3290 \mathrm{pg} / \mathrm{mL}$ was observed in a cohort of critically sick patients with a heart disease while that in the children with respiratory infection/illness was a significantly lower value of $17.4 \mathrm{pg} / \mathrm{mL}$ [11].

Studies done by Cohen et al and Koulouri et al in 2005 and 2004 respectively report similar findings that NT pro-BNP can differentiate between cardiac or pulmonary etiologies for children presenting with dyspnea or respiratory distress $[12,13]$.

Iacob et al enrolled 24 consecutive children with heart failure due to congenital heart diseases and dilated cardiomyopathy and measured their serum levels of NT pro-BNP and found a negative correlation with the ejection fraction of the left ventricle $(r=-0.165)$ [14]. Our study showed similar negative correlation between LVEF and NT pro-BNP levels although we have included all children with heart failure even without cardiomyopathy or underlying cardiac disease.

A study done by Mir et al in their study showed that NT pro-BNP levels were significantly higher in children with congestive heart failure than in control children, negative correlation with the ejection fraction $(r=-0.53)$ and a positive correlation with the clinical heart failure score $(r=0.74)$. [MIR] This is similar to the results obtained in our study which showed a 
significantly higher mean NT pro-BNP level value in those children with congestive heart failure which reflects the impairment of cardiac function in children with congestive heart failure [15].

Several studies have demonstrated that one third or more of patients presenting with CHF have normal left ventricular systolic function $[16,17,18]$. These patients, who most often have systemic hypertension, are believed to have heart failure due to diastolic dysfunction [17].

The limitations of this study include the assessment of only left ventricular ejection fraction on echocardiograph for diagnosing heart failure. Probably assessing other parameters of ventricular dysfunction will give a more accurate result. Further studies on the difference in NT pro-BNP levels in mild, moderate and severe ventricular dysfunction needs to be studied. Systolic and diastolic dysfunctions were not differentiated in this study.

\section{Conclusion}

This study showed that there is a significant difference in the NT pro-BNP levels of patients with cardiac failure and those with respiratory etiology and also a negative correlation between LVEF and NT pro-BNP levels. This proves that NT pro-BNP can independently predict low left ventricular ejection fraction and in turn heart failure. This method can be used to differentiate a cardiac failure from a respiratory pathology in patients presenting with dyspnea as it is a simple procedure not requiring the expertise of a cardiologist.

\section{What this study adds to existing knowledge?}

There are not many studies done showing the diagnostic value of NT pro-BNP in children. This study shows that although it is known that NT pro-BNP can be used as a marker for cardiac failure in adults, the use in children is limited. Values of NT pro-BNP have been researched but our study show a higher value can be obtained in cardiac patients than the published norms.

\section{Contribution of Authors}

Dr. Aswathy Rajan- Data collection, data analysis, manuscript drafting, editing

Dr. Ashvij Shriyan- Manuscript drafting, editing

Dr. Prem Alva- Data collection, data analysis, manuscript editing

Dr. Santosh Soans- manuscript editing

Funding: Nil, Conflict of interest: None initiated, Perission from IRB: Yes
Ethical Standards- The authors assert that all procedures contributing to this work comply with the ethical standards of the relevant national guidelines on human experimentation and with the Helsinki Declaration of 1975, as revised in 2008, and has been approved by the institutional committee A.J. Ethics Committee."

\section{References}

1. Hsu DT, Pearson GD. Heart failure in children: part I: history, etiology, and pathophysiology. Circ Heart Fail. 2009 Jan;2(1):63-70. doi: 10.1161/ CIRCHEART FAILURE. 108.820217.

2. Maher KO, Reed H, Cuadrado A, Simsic J, Mahle WT, DeGuzman M et al. B-Type Natriuretic Peptide in the Emergency Diagnosis of Critical Heart Disease in Children. Pediatrics, June 2008; 121 (6):e1484-1488. DOI: $10.1542 /$ peds.2007-1856.

3. Hospital stays, hospital charges, and in-hospital deaths among infants with selected birth defects-United States, 2003. MMWR Morb Mortal Wkly Rep. 2007; $56(2): 25-29$

4. Rusconi PG, Ludwig DA, Ratnasamy C, Mas R, Harmon WG, Colan SD, Lipshultz SE. Serial measurements of serum NT-proBNP as markers of left ventricular systolic function and remodeling in children with heart failure. American heart journal. 2010 Oct 1; 160 (4):776-83

5. Nir A, Nasser N. Clinical value of NT-ProBNP and BNP in pediatric cardiology. J Card Fail. 2005 Jun;11(5 Suppl):S76-80.

6. Lin CW, Zeng XL, Jiang SH, Wu T, Wang JP, Zhang JF, Ou YH. Role of the NT-proBNP level in the diagnosis of pediatric heart failure and investigation of novel combined diagnostic criteria. Experimental and therapeutic medicine. 2013 Oct 1;6(4):995-9.

7. Ootaki Y, Yamaguchi M, Yoshimura N, Oka S, Yoshida M, Hasegawa T. Secretion of A-type and Btype natriuretic peptides into the bloodstream and pericardial space in children with congenital heart disease. The Journal of thoracic and cardiovascular surgery. 2003 Nov 1;126(5):1411-6.

8. Fernandes BA, Maher KO, Deshpande SR. Cardiac biomarkers in pediatric heart disease: A state of art review. World journal of cardiology. 2016 Dec 26;8 (12): 719 . 


\section{Letter to Editor}

9. Ross RD. The Ross classification for heart failure in children after 25 years: a review and an age-stratified revision. PediatrCardiol. 2012 Dec;33(8):1295-300. doi: 10.1007/s00246-012-0306-8. Epub 2012 Apr 5.

10. Zhou FJ, Zhou CY, Tian YJ, et al. Diagnostic value of analysis of H-FABP, NT-proBNP, and cTnI in heart function in children with congenital heart disease and pneumonia. Eur Rev Med Pharmacol Sci. 2014;18 (10): 1513-6.

11. Das S, Chanani NK, Deshpande S, Maher KO. Btype natriuretic peptide in the recognition of critical congenital heart disease in the newborn infant. Pediatr Emerg Care 2012; 28: 735-738 [PMID: 22858747 DOI: 10.1097/PEC.0b013e3182624a12]

12. Cohen S, Springer C, Avital A, et al. Aminoterminal pro-brain-type natriuretic peptide: heart or lung disease in pediatric respiratory distress? Pediatrics. 2005 May;115(5):1347-50. DOI:10.1542/ peds.20041429

13. Koulouri S, Acherman RJ, Wong PC, et al. Utility of B-type natriuretic peptide in differentiating congestive heart failure from lung disease in pediatric patients with respiratory distress. PediatrCardiol. 2004 Jul-Aug; 25(4):341-6. DOI:10.1007/s00246-003-0578-0
14. Iacob D, Butnariu A, Leucuţa DC, Samaşca G, Deleanu D, Lupan I. Evaluation of NT-proBNP in children with heart failure younger than 3 years old. Romanian Journal of Internal Medicine. 2017 Jun 1;55 (2): $69-74$.

15. Mir TS, Marohn S, Läer S, et al. Plasma concentrations of $\mathrm{N}$-terminal pro-brain natriuretic peptide in control children from the neonatal to adolescent period and in children with congestive heart failure. Pediatrics. 2002 Dec;110(6):e76.

16. Lubien E, DeMaria A, Krishnaswamy $P$, et al. Utility of B-natriuretic peptide in detecting diastolic dysfunction: comparison with Doppler velocity recordings. Circulation. 2002 Feb 5;105(5):595-601.

17. Vasan RS, Benjamin EJ, Levy D. Prevalence, clinical features and prognosis of diastolic heart failure: an epidemiologic perspective. J Am Coll Cardiol. 1995 Dec; 26 (7): 1565-74. DOI: 10.1016/0735-1097 (95) 00381-9

18. Bonow RO, Udelson JE. Left ventricular diastolic dysfunction as a cause of congestive heart failure. Mechanisms and management. Ann Intern Med. 1992 Sep 15;117(6):502-10.

\section{How to cite this article?}

Aswathy Rajan, Shriyan Ashvij, Prem Alva, Santosh T Soans. Correlation between NT pro-b-type Natriuretic peptide and left ventricular ejection fraction in children presenting with Dyspnea- a prospective cohort study. Int J Pediatr Res. 2018;5(11):569-574.doi:10.17511/ijpr.2018.i11.04. 